Original Article

\title{
Effects of lumbar lordosis assistive support on craniovertebral angle and mechanical properties of the upper trapezius muscle in subjects with forward head posture
}

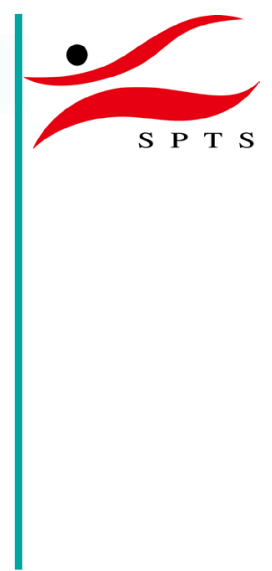

Jong-Hoon Moon, OT ${ }^{1) a}$, Jin-Hwa Jung, OT ${ }^{2) a}$, Suk-Chan Hahm, PT ${ }^{3)}$, Hwan-Kyung Oh, PT ${ }^{4}$, Kyoung-Sim Jung, $\left.\mathrm{PT}^{2}\right)^{* \#}$, Hwi-Young ChO, $\left.\mathrm{PT}^{5}\right)^{* \#}$

1) Department of Occupational Therapy, Graduate School, Gachon University, Republic of Korea

2) Department of Occupational Therapy, Semyung University: 65 Semyung-ro, Jecheon-si, Chungbuk 390-711, Republic of Korea

3) Department of Rehabilitation Standard and Policy, National Rehabilitation Research Institute, National Rehabilitation Center, Republic of Korea

4) Samsung Leports Bodywork Center, Republic of Korea

5) Department of Physical Therapy, Gachon University: 191 Hambangmoe-ro, Yeonsu-gu, Incheon 406-799, Republic of Korea

\begin{abstract}
Purpose] The aim of this study was to investigate the effect of lumbar lordosis assistive support (LLAS) on craniovertebral angle (CVA) and mechanical properties of the upper trapezius (UT) muscle in subjects with forward head posture (FHP). [Subjects and Methods] This study recruited 20 subjects with FHP. CVA and muscle tone, viscoelasticity, and stiffness of the UT were measured using Myoton in all subjects in a sitting position with LLAS and in a neutral sitting position. The order of measurements was randomized and the mean values were calculated twice. [Results] The sitting position with LLAS showed a significantly greater improvement than the neutral sitting position with regard to CVA and muscle tone, viscoelasticity, and stiffness of the UT. [Conclusion] We suggest that the sitting position using LLAS induces the maintenance of normal neck posture and a reduction in the muscle tone of the UT in the subjects with FHP.

Key words: Craniovertebral angle, Forward head posture, Lumbar lordosis assistive support
\end{abstract}

(This article was submitted Oct. 30, 2017, and was accepted Dec. 22, 2017)

\section{INTRODUCTION}

Forward head posture (FHP) is commonly detected in patients with a neck problem and a posture in which the head is placed anteriorly above the trunk ${ }^{1}$. FHP is common in people who work long hours in sitting position and has been associated with neck pain and decreased range of motion ${ }^{2}$. Moreover, this neck problem often causes psychological disabilities in daily living, such as discomfort or depression ${ }^{3}$.

Craniovertebral angle (CVA) of people with FHP is less than 51 degrees, and this is closely related to the neck pain ${ }^{4}$. Neck pain in these patients was found associated with increased muscle tone and hyperactivity of the upper trapezius (UT) muscle $^{5)}$. Thus, it is very important to prevent induction of FHP for a successful treatment of the neck pain. Prevention of

aThese two principal co-authors contributed equally to this work.

\#These two corresponding authors contributed equally to this work.

*Corresponding authors. Kyoung-Sim Jung (E-mail: jkspt@hanmail.net); Hwi-young Cho (E-mail: hwiyoung@gachon.ac.kr) (C2018 The Society of Physical Therapy Science. Published by IPEC Inc.

(c) (i) $\odot$ This is an open-access article distributed under the terms of the Creative Commons Attribution Non-Commercial No Deriva(C) ${ }_{\text {BY }}$ ND tives (by-nc-nd) License. (CC-BY-NC-ND 4.0: https://creativecommons.org/licenses/by-nc-nd/4.0/) 
FHP may help induce a correct posture and reduce the hyperactivation and hypertonus of the UT muscle.

Long sitting position reduces the lumbar lordosis, while the lumbar lordosis reduction produces FHP. This can cause back pain, because it increases the intra-pressure of inter-vertebral discs ${ }^{6}$. Therefore, the maintenance of lumbar lordotic curve during the sitting position is a key factor to prevent FHP and the UT fatigue.

Surface EMG is commonly used to objectify the UT problems. Surface EMG, however, does not directly reflect muscle mechanical properties, such as muscle tension ${ }^{7}$. Measurements using Myoton to quantify muscle mechanical properties, such as muscle tension, can be easily performed in clinical settings. Evaluation of muscle mechanical properties using Myoton is closely related to muscle activity generated by surface $\mathrm{EMG}^{8}$.

Andersson et al. reported that the use of lumbar support increased pelvic anterior tilt and the lumbar lordosis ${ }^{9}$. Recently, Ko et al. reported that the reduction of FHP in healthy elderly was greater in the sitting position with a lumbar roll support than in the neutral sitting position. These results suggest that the lumbar roll support induces upright lumbar lordosis during the sitting position ${ }^{10)}$. Yoo et al. found that the trunk muscle activity was different according to the application height of the lumbar support device, and authors suggested that this device was claimed to prevent musculoskeletal disorders ${ }^{11)}$. In another study, Horton et al. reported that applying a lumbar support roll to a chair could reduce FHP ${ }^{12}$. Previous studies, however, did not confirm the effect of the lumbar lordosis device on muscle properties of the $\mathrm{UT}^{9-12)}$. In addition, few studies on lumbar lordosis device for people with FHP were performed.

Thus, the purpose of this study was to investigate the effect of the lumbar lordosis assistive support (LLAS) on CVA and mechanical properties of the UT in subjects with FHP.

\section{SUBJECTS AND METHODS}

This study included 20 subjects with FHP among Gachon University staff and students. Subjects were included if they had a CVA of 51 degrees or less ${ }^{13}$. The following exclusion criteria were applied: any neck, shoulder, or back operative experience, or neurological signs in arms and legs. All subjects participated in the experiment with voluntary consent. The general characteristics of the subjects are presented in Table 1. This study was approved by the Gachon University Institutional Review Board (1044396-201708-HR-133-01).

All subjects were randomly placed in the neutral sitting position and in sitting position with LLAS. CVA and mechanical properties of the UT were evaluated in the sitting position. All measurements were performed by a single experimenter. LLAS (Chiropractic cushion, Balancecord Inc., Republic of Korea) used in this study is a tool that helps to maintain the posture of the lumbar lordosis during the sitting position. Additionally, it has a belt that fixes the subject on the chair. Once LLAS is fixed on the chair, the lordotic curve of the subject is fitted with LLAS comfortably. The application method of LLAS is as follows. The subjects sit up straight on the chair with two feet put on the floor. The LLAS was applied to L2-4 area to support lordotic curve.

CVA was measured in the sitting position using a digital camera and ImageJ software (National Institutes of Health, USA). The distance between the subjects and the camera was $80 \mathrm{~cm}$. The posture of the subjects allowed their feet resting naturally on the floor and both hands laying comfortably on the knees ${ }^{12)}$. The height of the chair was $45 \mathrm{~cm}$. The angle between the floor and the back of the chair was 90 degrees. Subsequently, the measurer attached the body markers to the external auditory meatus and the spinous process of the seventh cervical vertebra of the subjects. CVA was measured between the vertical line connecting the spinous process of the seventh cervical vertebra to a parallel line to the ground and the line connecting the spinous process of the seventh cervical vertebra to the external auditory meatus. The measurement was performed by one measurer, and the average value was calculated two times. In a previous study, the reliability of the test-retest of the CVA was $0.88-0.98^{14)}$.

Mechanical properties of the UT were measured using Myoton PRO (Myoton AS, Tallinn, Estonia). The mechanical properties of the UT were determined based on muscle tone $(\mathrm{Hz})$, stiffness $(\mathrm{N} / \mathrm{m})$, and viscoelasticity (logarithmic decrement of dampening oscillations). Measurements were performed with the subject in a sitting position on the chair in a right posture and in a quiet environment. All measurements were performed in a relaxed state with no force, and the postures were analyzed with the subject in the sitting position with LLAS and in the neutral sitting position. The measuring point of the UT was assessed based on the midpoint of the horizontal line connected from the acromion to the spinous process of the seventh cervical vertebra. The probe was measured perpendicular to the skin. In a previous study, the intra-class correlation coefficients of the UT measurements were $0.97-0.99^{15)}$.

All collected data were analyzed using SPSS 22 (IBM Corp., Armonk, NY, USA). The general characteristics of the subjects were identified through descriptive statistics. The normality of dependent variables was confirmed using the Shapiro-Wilk test. The differences in dependent variables were confirmed using a paired t-test. The significance level was set at $\alpha=0.05$. 
Table 2. Comparison of craniovertebral angle during the two sitting

\begin{tabular}{|c|c|c|}
\hline & Neutral & LLAS \\
\hline & Mean \pm standard deviation & Mean \pm standard deviation \\
\hline Craniovertebral angle & $46.82 \pm 1.46$ & $50.92 \pm 1.94^{*}$ \\
\hline
\end{tabular}

LLAS: lumbar lordosis assistive support.

$* \mathrm{p}<0.05$.

Table 3. Comparison of muscle tone, viscoelasticity, stiffness on upper trapezius during the two sitting

\begin{tabular}{lccc}
\hline & Neutral & & LLAS \\
\cline { 2 - 2 } & Mean \pm standard deviation & & Mean \pm standard deviation \\
\hline Muscle tone & $17.3 \pm 1.7$ & & $19.3 \pm 2.2^{*}$ \\
Viscoelasticity & $1.07 \pm 0.10$ & & $0.90 \pm 0.08^{*}$ \\
Stiffness & $375.4 \pm 58.5$ & $320.8 \pm 45.1^{*}$ \\
\hline
\end{tabular}

LLAS: lumbar lordosis assistive support.

$* \mathrm{p}<0.05$.

\section{RESULTS}

CVA in the sitting position with LLAS was significantly higher than that in the neutral sitting position $(\mathrm{p}<0.05$; Table 2$)$. Moreover, muscle tone, stiffness, and viscoelasticity of the UT in the sitting position with LLAS were significantly lower than those in the neutral sitting position $(\mathrm{p}<0.05$; Table 3$)$.

\section{DISCUSSION}

FHP was found to be associated with decreased CVA and change of lumbar lordosis. We used LLAS, which determines the appropriate curve of lumbar lordosis in the sitting position, to confirm the effect on CVA and the mechanical properties of the UT of the subjects with FHP. As a result, CVA while sitting with LLAS was significantly higher than that in the neutral sitting position, and mechanical properties of UT were significantly lower than those measured in the neutral sitting.

The first finding of the present study was that the CVA while sitting with LLAS was higher than that in the neutral sitting. This result is consistent with previous studies showing that lumbar lordosis affects FHP. Black et al. found that pelvic anterior tilting produces lumbar lordosis, which induces correct posture of the neck ${ }^{19}$ ). After applying LLAS in this study, the positive changes of mechanical properties of UT suggest that LLAS induces correct lumbar lordosis. Majeske et al. stated that the use of a lumbar support pillow in the sitting position can maintain sitting position effectively as it maintains the lumbar lordosis in place ${ }^{16)}$. Andersson et al. reported that the use of lumbar support increased pelvic anterior tilt and improved lumbar lordosis, which, in turn, would have a positive impact on the neck posture ${ }^{9}$. In addition, Ko et al. reported that sitting with lumbar roll support reduced FHP compared to the neutral sitting ${ }^{10)}$.

The second result of the present study is that the mechanical properties of UT were lower while sitting with LLAS than in the neutral sitting position. In this study, several mechanical properties of the UT, namely muscle tone, viscoelasticity, and stiffness, were evaluated. These three variables were reportedly found highly related to each other. The sitting position with LLAS showed a greater improvement in CVA, muscle tone, viscoelasticity, and stiffness of the UT than the neutral sitting position. This fact suggests that the reduction of FHP decrease muscle tone of the UT.

Increased muscle tone of the UT is a dysfunction of the shoulder, neck, and the muscles that compensate when proper movement does not occur ${ }^{15)}$. Computer workers who must work for long periods of sitting will have increased muscle activity of the UT resulting in lower muscle activity of the lower trapezius and serratus anterior. This eventually leads to an increase in the incidence of the neck and shoulder pain due to the imbalance of the shoulder joint and the increase in $\mathrm{FHP}^{17}{ }^{18)}$. Therefore, the application of LLAS during sitting position in this study may help reduce the incidence of shoulder and neck pain.

This study has several limitations. First, the sample size was small. Second, the subjects participating in this study were only people with FHP. Thus, the result cannot be generalized to all patients with neck pain. Third, this study only confirmed a short-term effects of LLAS. Fourth, we only identified the changes of mechanical properties of UT following LLAS application. In the future, the effect of LLAS should be clarified by supplementing these limitations.

In conclusion, this study was performed to investigate the effects of LLAS on CVA and mechanical properties of the UT of subjects with FHP. As a result, CVA in the sitting position with LLAS was significantly higher than that in the neutral sitting 
while the muscle tone of the UT was significantly lower in the sitting position with LLAS. The results of the study suggest that sitting with LLAS may have a positive effect on CVA and muscle tone of the UT in subjects with FHP.

\section{Conflict of interest}

No conflicts of interest.

\section{REFERENCES}

1) Shaghayegh Fard B, Ahmadi A, Maroufi N, et al.: Evaluation of forward head posture in sitting and standing positions. Eur Spine J, 2016, 25: 3577-3582. [Medline] [CrossRef]

2) Falla D, Jull G, Russell T, et al.: Effect of neck exercise on sitting posture in patients with chronic neck pain. Phys Ther, 2007, 87: 408-417. [Medline] [CrossRef]

3) Linton SJ: A review of psychological risk factors in back and neck pain. Spine, 2000, 25: 1148-1156. [Medline] [CrossRef]

4) Yip CH, Chiu TT, Poon AT: The relationship between head posture and severity and disability of patients with neck pain. Man Ther, 2008, 13: 148-154. [Medline] [CrossRef]

5) Fernández-de-Las-Peñas C, Cuadrado ML, Pareja JA: Myofascial trigger points, neck mobility and forward head posture in unilateral migraine. Cephalalgia, 2006, 26: 1061-1070. [Medline] [CrossRef]

6) Schomacher J, Farina D, Lindstroem R, et al.: Chronic trauma-induced neck pain impairs the neural control of the deep semispinalis cervicis muscle. Clin Neurophysiol, 2012, 123: 1403-1408. [Medline] [CrossRef]

7) Schomacher J, Erlenwein J, Dieterich A, et al.: Can neck exercises enhance the activation of the semispinalis cervicis relative to the splenius capitis at specific spinal levels? Man Ther, 2015, 20: 694-702. [Medline] [CrossRef]

8) Korhonen RK, Vain A, Vanninen E, et al.: Can mechanical myotonometry or electromyography be used for the prediction of intramuscular pressure? Physiol Meas, 2005, 26: 951-963. [Medline] [CrossRef]

9) Andersson GB, Murphy RW, Ortengren R, et al.: The influence of backrest inclination and lumbar support on lumbar lordosis. Spine, 1979, 4: 52-58. [Medline] [CrossRef]

10) Ko SH, Kim YS, Yoon BC: Influence of the lumbar spine adjustment using the lumbar roll support on head and neck posture in older adults. J Korea Contents Assoc, 2011, 11: 800-806. [CrossRef]

11) Yoo WG, Yi CH, Cho SH, et al.: Effects of the height of ball-backrest on head and shoulder posture and trunk muscle activity in VDT workers. Ind Health, 2008, 46: 289-297. [Medline] [CrossRef]

12) Horton SJ, Johnson GM, Skinner MA: Changes in head and neck posture using an office chair with and without lumbar roll support. Spine, 2010, 35: E542E548. [Medline] [CrossRef]

13) Kim KH, Kim SG, Hwangbo G: The effects of horse-riding simulator exercise and Kendall exercise on the forward head posture. J Phys Ther Sci, 2015, 27: 1125-1127. [Medline] [CrossRef]

14) Quek J, Pua YH, Clark RA, et al.: Effects of thoracic kyphosis and forward head posture on cervical range of motion in older adults. Man Ther, 2013, 18: 65-71. [Medline] [CrossRef]

15) Viir R, Laiho K, Kramarenko J, et al.: Repeatability of trapezius muscle tone assessment by a myometric method. J Mech Med Biol, 2006, 6: 215-228. [CrossRef]

16) Majeske C, Buchanan C: Quantitative description of two sitting postures. With and without a lumbar support pillow. Phys Ther, 1984, 64: 1531-1535. [Medline] [CrossRef]

17) Johnson GR, Pandyan AD: The activity in the three regions of the trapezius under controlled loading conditions--an experimental and modelling study. Clin Biomech (Bristol, Avon), 2005, 20: 155-161. [Medline] [CrossRef]

18) Ludewig PM, Cook TM: Alterations in shoulder kinematics and associated muscle activity in people with symptoms of shoulder impingement. Phys Ther, 2000, 80: 276-291. [Medline]

19) Black KM, McClure P, Polansky M: The influence of different sitting positions on cervical and lumbar posture. Spine, 1996, 21: 65-70. [Medline] [CrossRef] 